\title{
Referee's Influence on Competition Order in Dragonboat Race
}

\author{
Lei Luo \\ Hubei Business College \\ Wuhan, Hubei, China 430079
}

\begin{abstract}
Dragonboat sport, as one of traditional ethnic sports of our country, is well received by the whole nation, and various dragonboat races are carried out all the rage. And the referee, as the concrete implementer of dragonboat races, has significant influence on the competition order in races. This article adopts document literature method, expert interview method and logical analysis method to analyze the referee's influence on competition order and put forward the dragonboat referee's culture and development direction.
\end{abstract}

Keywords-dragonboat race; referee; competition order; influence

\section{INTRODUCTION}

Dragonboat race is a very prevalent traditional sport popular with people in our country. After the original national sports commission listed Dragonboat sport as national normal competition in 1984, organizations and institutions gradually perfected as well, with the vigorous promotion of Chinese Longzhou Association, Dragonboat sport boomed all over the country. The most visualized and acceptable form for people to contact Dragonboat is Dragonboat race. Only conducting Dragonboat race in order, will it be continuous and wonderful so that the audiences have more interest in Dragonboat and it will be more easy to accept and promote; otherwise, Dragonboat will has low ornamental value, and the audiences will be disappointed even disgusted, resulting in the promotion of Dragonboat is in passive condition.

Referee, as the organizer of Dragonboat race, directly determines the performance of the competition, and referee works throughout the competition, hence, whether the competition conducts in order, is inseparable with referee work to a great extent. If the referee does a good job, it will convince the sports team and audiences, then the competition will carry out as planned, so the scene is wonderful; otherwise, if the referee does not work that well, then not only the competition schedule may be hampered, but also the sports team and audiences may seize the chance to cause trouble, even evolve into incident of violence, and give rise to negative influence. In this article, it adopts document literature method, expert interview method and logical analysis method to study the negative influence due to referee fault on competition order, and put forward aimed suggestions, raise opinions of improving the referee work in Dragonboat race, so as to provide theory basis for the culture of Dragonboat referee, and better promote the development of Dragonboat.

\section{RESULT AND ANALYSIS}

There are lots of reasons causing Dragonboat race conducts in no order, such as arrangement of competition site and logistics service after competition etc. but referee work is one of the factors vulnerable to blame and focus. Chinese Longzhou Association determines to take building referee team as key and breakthrough of promoting Dragonboat sport all the time. Especially in the two competitions of national Dragonboat public competition and Chinese Dragonboat competition, it gives more strict requirements of referee work. Hence, in comparison with other project, there are less faults existing in referee work of dragon work. However, it is known to us as well that referee work is still less than satisfactory in local Dragonboat race, which causes that the competition is not smooth and the scene is not in order, even group violent incidents happen.

\section{A. Influence of Referee Communication on Competition Order}

Smooth communication is the foundation of carrying out group activities. Good communication can discover signs of disordered competition in time, so that it can dispose the problem as soon as possible and stifle in the cradle. On the contrary, self-centered communication in bad attitude may sharpen the contradiction, resulting in factors which should have been tiny and unable to cause mess become group events.

1) Communication among referees: Generally, referee teams are equipped with interphones for convenience of contacting in dragon race. However, minority referees complete their team's work as their own time nodes without communication with other teams forced by the pressure at site, which results in work disjunction of each referee team and brings huge pressure to other referee teams, even causes suspension of competition. For instance, the check-up referee leads the teams to board boat before the equipment referee well preparing, the start point referee neglects the terminal referee's request of issuing a second order, and the check-up referee dose not report the draw result to other referee teams in time etc.

2) Communication between referees and sports team: Since the scope of competition site for Dragonboat race is large and special, the athletes are not able to concentrate as easily as other project, and the athletes' levels are uneven, even some of them are not familiar with the competition rules 
and may have different views of understanding on referees. And it requires that referees should communicate with the sports teams with nice attitude and patient, as well as disclose information for sports teams' convenience of understanding for information symmetry. Thus the athletes can understand the referee, actively cooperate with referee work, then the competition can be successfully completed as given time nodes in amicable atmosphere. While if referee communicate with athletes in bad attitude, and blindly blame them, it may easily cause opposition between sports team and referee and the athletes may do anything to referee in opposition, even delay or refuse to board on purpose or boat back not in time etc.

3) Communication between referee and audiences: Dragon referees compose of water referee and bank referee, especially the bank referee is more close to audiences, due to the fact that most audiences are not familiar with competition rules, hence, they more focus on the atmosphere of the race, as for the competition rules, they are not in their consideration, hence, sometimes minority audiences may make certain influences on referee work. It requires that referees should pay attention on their own images while contacting the audiences, and guide audiences to watch competition in a civilized manner from professional view, and under allowable conditions, it may disclose proper information of competition to meet audiences' curiosity, and for problems difficult to solve, it shall actively ask for committee's help. While referees who are not conscious of their images, think of they being a notch above the others, it may cause audiences' dislike, even result in conflicting with the audiences, and the audiences block the competition site, impact the work area of referee as well as siege referees etc.

4) Communication between referee and organizing committee: Organizing committee is the specific organizer of the competition, while referee is the concrete implementer of the competition, hence, referee shall work under the guidance of the committee. The committee masters more information and resources than referee, and the referee, especially the chief referee shall keep in good and active touch with the committee for competition information collection. In competition, matters beyond one's responsibility shall be actively reported to the committee in time, and matters which are not proper to solve in identification of referee shall be actively asked for committee's help, hence, each performing its own functions will be good for carrying out the competition in order.

\section{B. Influence of Referee's Personal Ability on Competition Order}

Dragonboat sport is one of the projects having most athletes, most complex site and most audiences. And the complex work environment and enormous pressure form extremely great pressure to the referees' personal ability.

1) Language expressive ability: Language is the basis of communication. The language expressive ability of referees firstly is reflected on their mastering of mandarin: China has vast territory and big differences of local dialects correspondingly, and the athletes and judgments in dragon match are from all over the country. Proficiency in mandarin is good for referees to express and better communicate with athletes without gap. Secondly, from the view of language precision: refined and accurate expression is more good for the opposite to understand its meaning, while verbose and equivocal expression may cause the opposite cannot get the point, or misinterpret the meaning due to different positions, resulting misunderstanding. In addition, it reflects on the mastering of English language: with the internationalization of Dragonboat sport, more and more foreigners participates, correspondingly the referees have more opportunities to work aboard. On this basis, it seems to be more important for the referees to master good English language ability.

2) Ability of site organization: Due to most athletes and complex site the Dragonboat referees facing, it requires they having good organizing ability. Before competition, they shall evaluate the situation at site, and lay down their plans of referee points, so that they can meticulously organize athletes to pass the point as planned during competition. Favorable organization ability can make the athletes compete as planned rapidly, scientifically and effectively, and make the whole scene look in perfect order and feast the audiences' on the competition. While in case of low organization ability, it may cause the athlete cannot find their team, the team cannot find the referee or the referee cannot find the site, resulting in stage wait due to competition delay and making a negative impression to the audiences.

3) Ability of making immediate response: Due to complex circumstance and excessive personnel, it will cause more random events and the more events the more it examines the referees' ability to making immediate response and disposing sudden situations in complex circumstance. And proper response and in-time disposition will convert the chaos back to normal, make the competition order in controlled scope; in case of poor ability of making immediate response, disposing incidents not in time or decisively, it may cause chaos even safety accident. And if boat collision is not disposed in time, it may result in drowning or missing accidents.

\section{Influence of fairness of Referee Work on Competition Order}

The first priority of referee, in any competition, is fairness. Fairly treating every sports team in refereeing process is security of guaranteeing the competition carrying out smoothly. Every judgment of referee has the direct bearing on the practical interest of both teams, if lack of justice and fairness, certainly it will cause dissatisfactory and confliction of sports teams and audiences, even it may cause confliction between the sports teams and referee, confliction between both sports team as well as confliction between audiences and referee.

1) Intended preference of referee: Basically no referee is full time who is active in Dragonboat sport, hence certainly there will be various relations, especially some units the referee belonged still participate in the competition. By thus case, the referee will actively prefer the team of own unit on purpose, and benefit the team within permissible scope of the rules, which is beneath the professional ethics and influences the fairness of the competition.

2) Refereeing scale of referee: As a referee, it is the first priority of familiar with competition rules and referee law. 
However, different people have different understandings on rules, so are their characteristics and working methods, hence, every referee masters different scale in refereeing process. Especially the qualification examination at roll call, false start determination at starting point and water distance of track, if different referees mater different scales in same competition, it may cause sports teams query the fairness of referee work.

3) Tools use of referee: Currently most referee work of Dragonboat competition is completed manually, inevitably tools are required in refereeing process, such as stopwatch, computer, siren and yacht etc. if referees are not familiar with using these tools, it may delay the opportunity, which may result in misunderstanding to sensible sports team due to delayed time, and the team may think that the referee has different standards, so as to query the competition result and further cause chaos.

4) Work flow of referee: Every point in Dragonboat competition the referee has its own work flow, but in practical operation, the referees have different preciseness in process of implementing the flow. Especially under situation of excessive race times and delayed time, minority referees will simplify or omit the work flow which should have been executed strictly, such as qualification examination, roll call at the starting point and arrival whistling at the end point. Which may be utilized by intended sports team to cause situations of imposture, mess tracks and ranking determination query etc. and then it will influence the fairness of the competition and cause disorder.

\section{COUNTERMEASURES AND SUGGESTIONS}

\section{A. Strengthen Business Training of Referees}

Referee work is complex, which requires referee mastering various knowledge; and the referee work is long term work, which requires referee continuously studying, hence, strengthening business training of referee is necessary. Firstly, it shall conduct multiple trainings of competition rules and referee law to make the referee master the rules and understand the meaning of rules as well as standard refereeing scale; secondly, conduct training of professional ethics to make sure that all the referees can keep their professional ethics strictly free from fault; thirdly, conduct the training of language ability to promote their own communication abilities for precisely expressing their meaning; and at last, conduct training of tools use to make referees familiarly use any tools related in refereeing process.

\section{B. Strengthen Echelon Building of Referee Talents}

In Dragonboat sport, there are both experienced referees active in competition for long term and new referees rarely appearing at competition, hence, due to different refereeing experiences, there will be different personal abilities. In order to make up the gap, it requires strengthening the echelon building of referee talents, so as to make the experienced referees pass their experiences to the new ones and play their positive roles. Therefore, the referees of Dragonboat will have no fault, and the good tradition can pass the flame.

\section{Persist System of Double-Check Evaluation and Supervision on Referee}

In the Rules for the Implementation and Management of Dragonboat Referee issued by the Chinese Longzhou Association this year, it clearly states the system of monitoring and evaluation on Dragonboat referee, and there also is double-check system of referee in previous referee management. Carrying out these systems can make the referees have self-study pressure, bonding them in system to promote their autorhythmicity. Successfully developing of the competition, the systems of double-check, evaluation and monitoring must be carried on all the time.

\section{Strengthen Culture of Referees' Service Consciousness}

Change the inherent thoughts of the referee and promote its service consciousness for the competition and sports team. The work of referee is to guarantee the competition to progress smoothly, so the sports teams hope, hence, it should not be opposite relation between the referee and sports team. Enhance the referees' service consciousness will make them correct attitude, focus on their own work ways, and based on same objective, the competition air will be harmonious with joint effort.

\section{CONCLUSION}

Referee work is the basic guarantee of carrying out competition smoothly. With the development of Dragonboat sport today, the referee needs to cherish the original intention of inheriting national tradition, actively enhance their own level, strictly obey the referee rules, fairly treat every Dragonboat race they participating and do their best to carry out the competition scientifically in order, feast the eyes of the audiences and athletes and make contribution to the sound development of Dragonboat sport.

\section{REFERENCES}

[1] Xu Jusheng, Yu Hanqiao. Advanced Course of Dragonboat Sport [M] Beijing: China Electric Power Press, 2015

[2] Chinese Longzhou Association. Rules for the Implementation and Management of Dragonboat Referee (trial) [EB/OL].http://dragonboat.sport.org.cn, 2016

[3] Chinese Longzhou Association. Rules for the Appointment and Supervision of Referee of Chinese Longzhou Association (trial) [EB/OL].http://dragonboat.sport.org.cn, 2015

[4] Xue Long, Zhang Kun. Influence of Referee on Football Competition Order [J]. Journal of Physical Education, 2003(7)

[5] Li Fan, Li Dongbo. Investigation and Analysis on the Current Situation of Track and Field Referees in Heilongjiang Province [J]. Journal of Harbin Institute of Physical Education, 2010(8)

[6] Xin Mengxia. Research on the Current Development Situation and Countermeasures of "Hubei Dragonboat Competition" [J]. Traditional Ethnic Sports, 2014(7)

[7] Wang Tingting. Research on the Developing Situation and Countermeasures of Athletics Dragonboat Sport Based on SWOT Analysis [D]. Beijing Sport University, 2010

[8] He Yingfeng. Analysis on International Promotion of Dragonboat Sport under New Form [J]. Heilongjiang Science and Technology Information, 2011(35) 
[9] Zhou Hua. Research on the Study Situation and Development Countermeasures of "Dragonboat Sport" in China [J]. Journal of Chifeng University(Natural Science Edition), 2009(5). 